Review

\title{
Update on TB Vaccine Pipeline
}

\author{
Carlos Martin 1,2,3,*®D, Nacho Aguilo ${ }^{1,2}$, Dessislava Marinova ${ }^{1,2}$ and Jesus Gonzalo-Asensio 1,2 (I) \\ 1 Grupo de Genética de Micobacterias, Microbiología, Facultad de Medicina Universidad de Zaragoza, \\ 50009 Zaragoza, Spain; naguilo@unizar.es (N.A.); tbvacman@unizar.es (D.M.); jagonzal@unizar.es (J.G.-A.) \\ 2 CIBERES Enfermedades Respiratorias, Instituto de Salud Carlos III, 28029 Madrid, Spain \\ 3 Servicio de Microbiología, Hospital Universitario Miguel Servet, 3ISS Aragón, 50009 Zaragoza, Spain \\ * Correspondence: carlos@unizar.es
}

Received: 10 March 2020; Accepted: 31 March 2020; Published: 10 April 2020

\begin{abstract}
In addition to antibiotics, vaccination is considered among the most efficacious methods in the control and the potential eradication of infectious diseases. New safe and effective vaccines against tuberculosis (TB) could be a very important tool and are called to play a significant role in the fight against TB resistant to antimicrobials. Despite the extended use of the current TB vaccine Bacillus Calmette-Guérin (BCG), TB continues to be transmitted actively and continues to be one of the 10 most important causes of death in the world. In the last 20 years, different TB vaccines have entered clinical trials. In this paper, we review the current use of BCG and the diversity of vaccines in clinical trials and their possible indications. New TB vaccines capable of protecting against respiratory forms of the disease caused by sensitive or resistant Mycobacterium tuberculosis strains would be extremely useful tools helping to prevent the emergence of multi-drug resistance.
\end{abstract}

Keywords: BCG; tuberculosis vaccines; TBVI; EDCTP; IAVI; CTVD

\section{Introduction}

To date, vaccines have been able to overcome the evolution of antibiotic-resistant strains, which makes vaccination one of the most cost-effective measures for fighting infectious diseases [1]. Mycobacterium tuberculosis (Mtb) is included as 'critical' in the WHO priority list of research and development for new antibiotics effective against current resistant strains of tuberculosis (TB) [2]. Concurrently, new efficacious vaccines will be a very important tool to fight antimicrobial resistant TB (AMR TB) and are called to play an important role against this serious health issue [1].

The potential of using TB vaccines to combat AMR TB has generally been undervalued. This could be partly due to the lack of integral efficacy of the present vaccine Bacillus Calmette-Guérin (BCG) failing to reduce the numbers of TB cases, which makes new efficacious vaccines against respiratory forms of TB a critical necessity to help combat AMR TB. Currently, one of the biggest threats in TB is the emergence of multidrug-resistant (MDR) $M t b$ strains, resistant to isoniazide and rifampicin, and extensively drug-resistant (XDR) strains, resistant to at least four of the core anti-TB drugs. In 2018, half a million people were diagnosed with MDR-TB and is estimated that fifty million people were infected with MDR Mtb strains, creating a reservoir for future cases of active TB making treatment extremely difficult [2]. AMR poses a threat in TB; both the World Health Organization (WHO) and International Centres for Disease Control and Prevention (CDC) have expressed concern about antibiotic treatments for TB. New TB vaccines are necessary to complement existing and in-development pipeline TB treatment and diagnostic strategies. Considering there has been no evidence to suggest that molecular mechanisms of drug resistance in $M t b$ could affect immune control susceptibility, it is likely that vaccine efficacy against MDR-TB and drug-sensitive TB will be equivalent [3].

In the present work we review the current use of BCG and the non-specific effect against other pathogens and we summarized the diversity of new TB vaccine candidates in clinical trials and 
their indications. We discuss the need to keep the pipeline of new TB vaccine candidates and the current clinical trial designs employed in the field for efficacy determination of new TB vaccines which include designs for prevention of infection (POI), prevention of disease (POD), and prevention of recurrence of TB (POR), or trials for evaluating the therapeutic effect of TB vaccine candidates when applied in combination with current $\mathrm{TB}$ drug regimens with the aim to shorten duration of treatment. The pipeline includes new prime TB vaccines for administration at birth, which are expected to protect better than BCG, and for use in adolescents and adults, as revaccination strategies in individuals previously vaccinated with BCG at birth, as per WHO Preferred Product Characteristics (PPC) for new TB vaccines [3,4]. Novel TB vaccine strategies, which are safe and effective, are imperative in the global efforts to halt dissemination of drug-sensitive and AMR TB [3].

\section{BCG the Current TB Vaccine in Use}

Despite its variable efficacy against respiratory forms of TB, BCG remains the only marketed vaccine in use against TB, with more than $90 \%$ coverage in countries with high TB incidence [5-7] (Figure 1). BCG is an attenuated vaccine derived from Mycobacterium bovis, the etiologic agent of TB in cattle [8]. BCG was first introduced in clinic almost a hundred years ago, when in 1921 it was administered orally to a baby whose mother had died of TB the day after her birth. The baby showed no adverse effects to BCG vaccination and, more importantly, did not develop TB. Between 1921 and 1926 more than 50,000 children were vaccinated. Mortality among vaccinated children was $1.8 \%$, compared to a mortality greater than $25 \%$ in unvaccinated children, showing its effectiveness in reducing infant mortality, not only due to TB if not due to other respiratory diseases [9]. Today we know that the main cause of attenuation of BCG is due to the loss of Region of Difference 1 (RD1) associated with the loss of the virulence factor of the secreted immunodominant antigen of $6 \mathrm{kDa}$ (ESAT-6) [8].

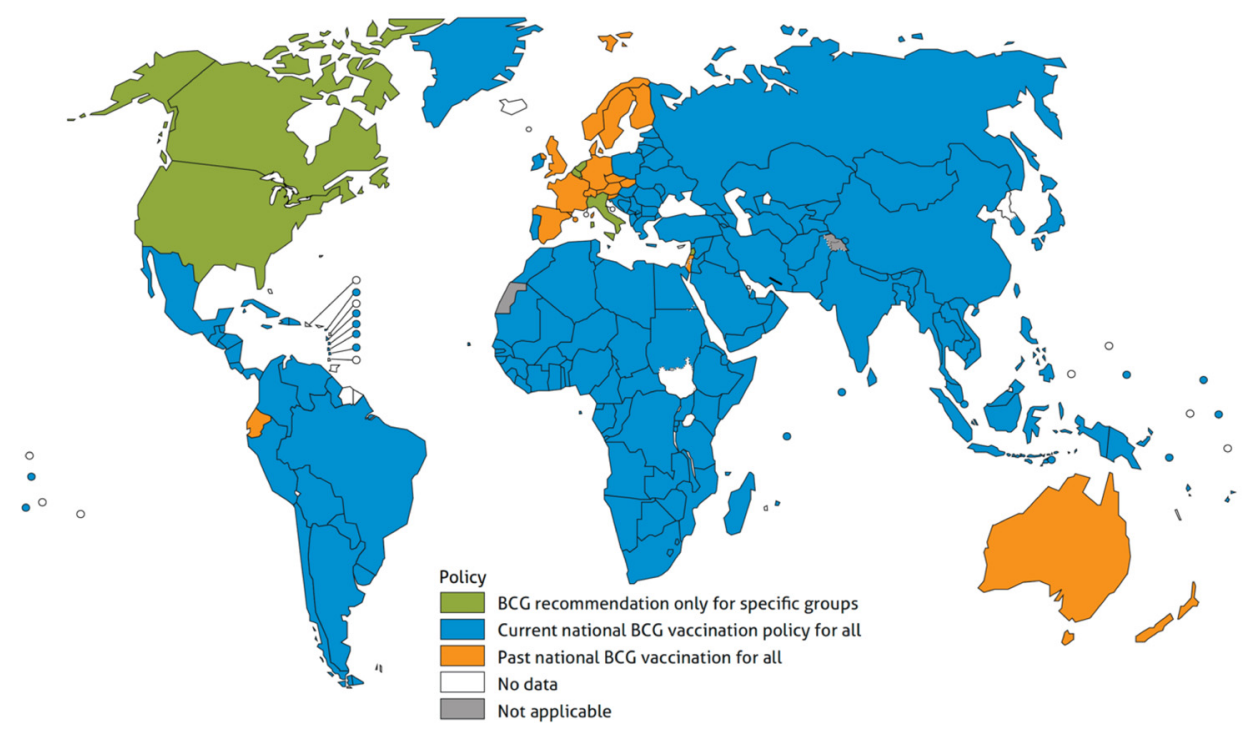

Figure 1. Bacillus Calmette-Guérin (BCG) vaccination coverage by country. Data from the World Health Organization about the BCG coverage in each country [10,11]. A total of 113 countries reported coverage of at least $90 \%$ [2].

In 1976, WHO established the Expanded Program on Immunization (EPI) to ensure universal access of mothers and infants/children to routinely recommended infant/childhood vaccines. Initially, there were six vaccine-preventable diseases included in the EPI: TB, poliomyelitis, diphtheria, tetanus, pertussis and measles. Intradermal vaccination with BCG at birth has been included in the WHO EPI, resulting in more than four billion vaccines administered worldwide to date and approximately 200 million doses given each year. Depending on strain and manufacturer of BCG, concentration of live bacteria in vaccine vials can range between 50,000 to three million per dose $[12,13]$. BCG vaccination is 
recommended as part of national childhood immunization programmes according to a country's TB epidemiology. In 2018, BCG vaccination was reported for 153 countries and 113 of these countries reported at least $90 \%$ BCG coverage $[2,10,11]$ (Figure 1).

Today, BCG vaccination is recommended by the WHO in all newborn infants in countries where TB incidence is high. In countries where the incidence of TB is not high, BCG is recommended if the child is continually exposed to TB patient who does not respond to treatment and patient's separation is not possible, or when the child is continually exposed to a patient who has infectious pulmonary TB caused by MDR or XDR strains [14]. The recommendation of BCG vaccination for adults in endemic areas with high exposure to resistant TB remains controversial. Considering the potential risks of anti-TB treatment failure and complications related to BCG vaccination of immunocompromised individuals, administration of BCG could be recommended in unvaccinated individuals, tuberculin-negative or interferon gamma release assays (IGRA)-negative individuals exposed to MDR TB. Thus, trials evaluating protective efficacy of BCG in the context of exposure to MDR TB in adults are needed [14].

Commercial BCG is not a unique product as different formulations exist in terms of BCG strain, composition and/or dosage. Currently, six BCG strains are used worldwide in international immunization programs: BCG Pasteur 1173 P2, BCG Danish1331, BCG Glaxo 107, BCG Tokyo 172-1, BCG Russia-I and BCG Brazil [15]. These different BCG strains in use [16] exhibit different characteristics of attenuation and protection in animal models [17]. The genomic analysis of BCG sub-strains shows multiple differences, including other deletions other than RD1 that contribute to phenotypic variations between them [17], with clear attenuation differences but without being shown to contribute to differences in their efficacy [5]. A recent systematic head-to-head comparison study of BCG vaccine formulations demonstrated marked variations in content of viable mycobacteria which correlated with age-specific induction of cytokines in vitro [13]. According to the same study, these differences in viability possibly contribute to an observed formulation-dependent activation of innate and adaptive immune responses that could account for the variable protection observed in clinic [13].

\section{Nonspecific Effects of BCG}

One of the reasons why BCG is still used universally in middle and low income countries is because numerous studies indicate that similar to other live attenuated vaccines in use today, BCG have additional beneficial effects on the initially intended protection against TB [18-20]. Neonatal BCG is able to induce a strong Th1 cytokine response shown to enhance immune responses to other infant vaccines of the EPI [21]. In countries of low TB endemicity, BCG administration at birth has been related to reduction of childhood hospitalizations due to unrelated respiratory infections and sepsis [22,23]. In addition, there is emerging evidence that BCG may induce nonspecific resistance (T- and B-cell dependent) to other pathogens [20], which should be taken into consideration for AMR TB.

The non-specific beneficial effects ascribed to BCG have been attributed to the vaccine's ability to functionally and epigenetically reprogram innate immune cells, such as monocytes, macrophages, and NK (natural killer) cells, a process termed 'trained immunity' [24]. In human monocytes, BCG induced trained immunity has been attributed to the induction of metabolic pathways, which are regulated by epigenetic mechanisms at the level of chromatin organization [25]. In this context, future clinical trials could provide insight on the potential therapeutic role that modulation of these pathways may have during vaccination [26]. A recent large, multinational study conducted in sub-Saharan Africa suggests association of BCG vaccination with a reduced risk of malaria in children under the age of 5 years $[27,28]$. If these results are corroborated, they would denote that timely BCG vaccination could aid the global efforts to decrease malaria burden, including resistant forms of the disease.

To date, the preclinical and clinical down-selection process of new TB vaccine candidates has employed BCG as the reference gold standard comparator, because of its well-established safety profile $[29,30]$. Today, WHO also encourages incorporation of the nonspecific beneficial effects ascribed to BCG's ability to induce trained immunity in the design and development of novel TB vaccine candidates, especially those intended for BCG-replacement. 


\section{New TB Vaccine Candidates from Discovery to Clinical Trials}

The most effective licensed vaccines against different infectious diseases confer protective immunity by /inducing neutralizing antibodies. Whereas, while for other diseases, such as HIV, malaria or TB, a strong response of cellular immunity is necessary, and we don't have correlated protection that could anticipate the efficacy of a new vaccine candidate [31]. The last 20 years have seen important breakthroughs in TB vaccinology, ranging from novel adjuvant systems or viral vectors for intracellular antigen presentation to advanced genetic engineering techniques for rational attenuation of $M t b$. All these advances in vaccinology have led to the development of new TB vaccine candidates including novel prime and prime-boost regimes that could reach all age groups and TB population spectrums. The biology behind the host immune responses to $M t b$ are not yet understood [32,33]. Developing a protective vaccine requires not only finding the right antigens, but also activating the right ratio of protective and suppressive immune cells against these pathogens. In Douglas Young's words, "we need efficacious vaccines to understand immunology of TB" and we need "immunology studies to design a good TB vaccine".

Given the lack of BCG protection against respiratory forms of $\mathrm{TB}$, in the last 20 years an enormous effort has been made in the research and development of new vaccines against TB. In the discovery phase, thousands of potential candidates were identified, of which hundreds have passed to preclinical evaluation in animal models and only a little more than a dozen have happened to be tested in early clinical studies in humans to date. There are different stages that each vaccine candidate needs to perform, including early first-in-human Phase I, then Phase II, and finally Phase III clinical trials, to reach marketing authorization. In the United States, for nearly two decades former Aeras (now International AIDS Vaccine Initiative, IAVI) [34], supported by the Bill and Melinda Gates Foundation, were dedicated to the discovery of new TB vaccine candidates. At the end of 2018, Aeras transferred its preclinical assets and clinical programs, biorepository, clinical staff, funding and other assets to International AIDS Vaccine Initiative (IAVI) [34]. In Europe, the research promoted by the different European Commission (EC) Framework Programs has made possible for hundreds of candidates to pass to preclinical evaluation of which several are currently in clinical trials [35]. In 2008, thanks to the EC funding programmes, the European TB Vaccine Initiative (TBVI) [36] was founded integrating at least 50 R\&D consortium partners from the public (academia) and private (industry) sectors. TBVI is a non-profit product development partnership that facilitates the discovery and development of new, safe and effective TB vaccines and biomarkers that are accessible and affordable for global use. Clinical trials of a small number of selected TB vaccine candidates are supported by IAVI with funding sources from US NIH funding programmes and by the European and Developing Countries Clinical Trials Partnership (EDCTP) [37].

Given that the protection of BCG administered intradermally shows such limited results of efficacy, new studies changing the routes of administration of BCG have demonstrated encouraging results in non-human primates (NHP) applying BCG by the intravenous (IV) route against virulent $M$. tuberculosis challenge [38]. Although the difficulties of using the IV route for mass vaccination campaigns, the efficacy results demonstrating ability of IV BCG to substantially limit $M t b$ infection in highly susceptible rhesus macaques could have important implications in the preclinical evaluation of new candidates, as it could provide a prototype for identifying immune biomarkers and mechanisms of vaccine-induced protection against TB. The respiratory administration of BCG has demonstrate to be very promising by conferring very good immunity and protection in NHP [39]. If these results are confirmed in clinical studies, the aerosol route could be considered a possible universal vaccination route for BCG and new TB vaccine strategies.

Other live attenuated vaccines in preclinical testing fuelling the TB pipeline include the search for new candidates based on recombinant BCG has shown very promising results in preclinical animal models. On one hand, deletion of zmp1 gene improves BCG-mediated protection in guinea pigs against TB [40] and on the other, inclusion of virulence genes, such as the RD1 region, genetically modified so as to not increase the virulence of BCG [41,42]. 


\section{TB Vaccine Candidates in Clinical Trials}

As we previously mentioned, the lack of immune marker(s) for prediction of vaccine-elicited protection makes finding effective vaccines against TB extremely challenging, as it requires long and expensive efficacy trials with thousands of volunteers (Phase IIb proof-of-concept trials and Phase III efficacy trials) in endemic countries with a high incidence of TB after obtaining robust safety and immunogenicity data in previous trials with tens (Phase I) and then with hundreds (Phase II) of volunteers [32] (Figure 2).

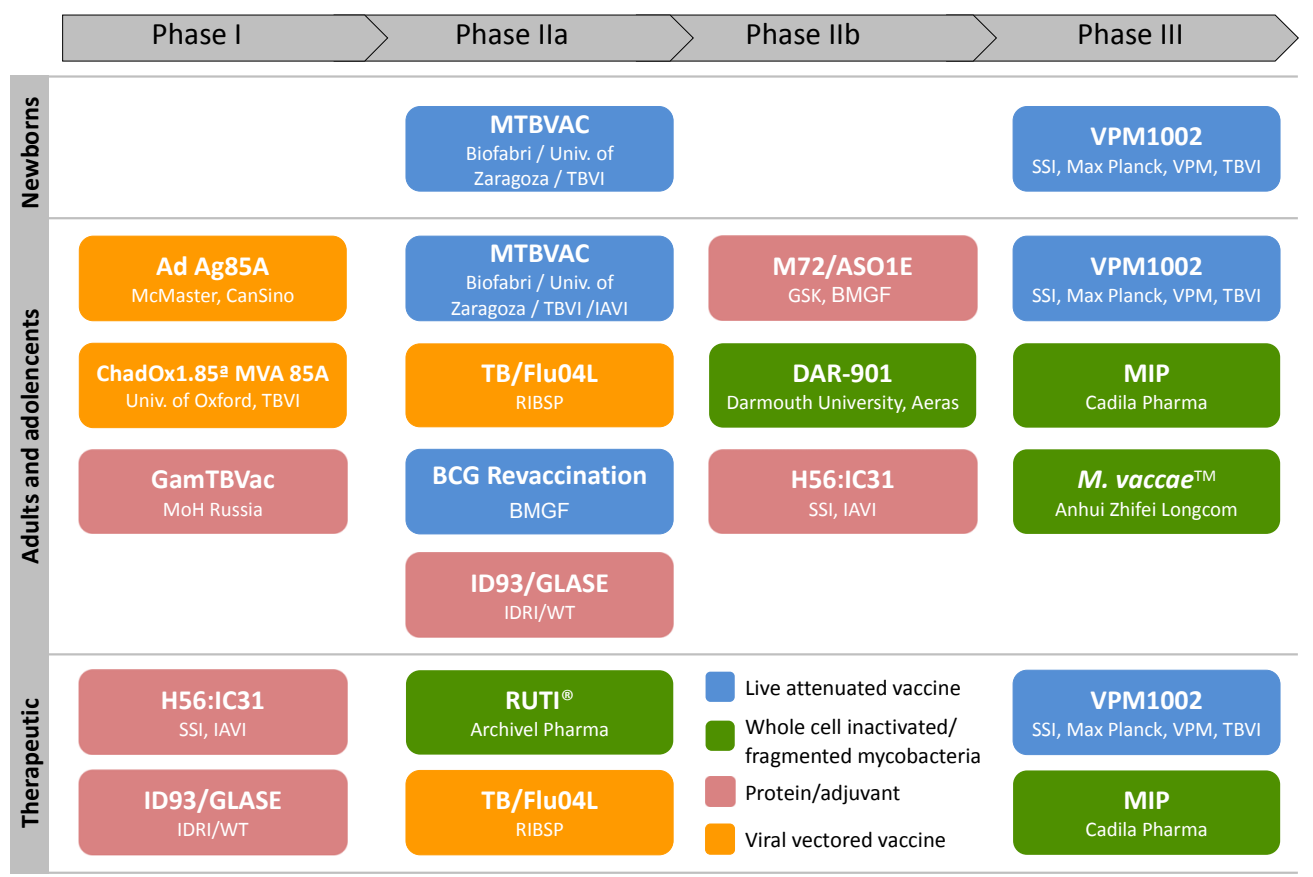

Figure 2. Tuberculosis (TB) vaccine candidates in the pipeline of clinical trials. The diagram shows the advance in clinical trials of the 14 vaccine candidates coloured according to each vaccine strategy.

Unlike HIV and malaria, twenty years ago there was no new candidate for a vaccine against TB in clinical trials. After more than 10 years of previous clinical trials, in 2012 MVA85A was the first candidate since BCG in 1921 to enter efficacy evaluation as preventive TB vaccine in infants [43,44]. MVA85A vaccine was tested in a double-blind, placebo-control Phase IIb efficacy study for its ability to increase (or boost) the immunity in healthy infants HIV-uninfected (aged four to six months) recently vaccinated with BCG at birth, living in a highly endemic region for TB (Worcester, South Africa). A total of 2797 children were vaccinated (1399 with MVA85A and 1398 with a placebo) and followed up every three months for more than three years. Results showed that 32 children $(2 \%)$ of the 1399 in the MVA85A arm, were diagnosed with TB and 39 children (3\%) of the 1398 vaccinated with $\mathrm{BCG}+$ placebo. The difference between the two groups was not significant and the interpretation was absence of efficacy of MVA85A. For the TB vaccine scientific community, this study resulted highly informative, and it was a great step forward in the research of new vaccines paving the way for new TB vaccine efficacy studies. The Worcester study was coordinated by the South African TB Vaccine Initiative (SATVI) and the site is highly prepared for testing new promising TB vaccines. Years after this study, the scientific community continues learning and drawing conclusions about the immunology of the disease. After three years of follow-up of the children in the study, the QuantiFERON (QFT) and the risk of disease were studied by SATVI and Oxford University teams [45]. The extended immunogenicity analysis of the trial data showed that both children that remained QFT- (less than $0.35 \mathrm{IU} / \mathrm{mL}$ ) or those who became QFT + (with less than $4 \mathrm{UI} / \mathrm{mL}$ ) had a lower risk of developing TB as 
compared to QFT + children with more than $4 \mathrm{IU} / \mathrm{mL}$, whose risk of TB disease is high. This result may accelerate studies with other vaccines [45].

Current efficacy trials of new prophylactic TB vaccines attempt to measure either the prevention of infection against $M t b$ (POI), or the prevention of acquiring TB disease (POD) and the prevention of recurrent TB disease (POR) [46]. POR trials evaluate therapeutic vaccines administered as an adjunct to drug treatment to increase the effectiveness and shorten the duration of TB treatment in patients undergoing or completing TB treatment for active disease. The efficacy endpoints of POR trials are prevention of reactivation of existing infections and/or prevention of disease due to new infections. Considering lack of correlates of protection, POD trials provide the most reliable endpoint of vaccine efficacy and acceptance for Regulatory authorities.

In newborns, new TB vaccines should provide evidence of significant superiority over BCG. Global vaccination strategies targeting adolescents and adults would preferably include individuals with and without pre-existing $M t b$ infection, thus avoiding use of IGRAs which are expensive and can interfere with vaccine-induced immune results. In addition, testing candidates in both uninfected and infected individuals in future trials could avoid risks of excluding potentially efficacious candidates in the pipeline against infection but which lack efficacy in IGRA-positive individuals [47].

New TB vaccine candidates today in clinical trials could be divided into whole cell vaccines and subunit vaccines. Whole cell vaccines include live mycobacterial vaccines derived from live attenuated $M t b$ strains, $M$. bovis BCG or recombinant BCG and killed mycobacterial vaccines that could be formulated from other saprophytic mycobacterial species or $M t b$ [48] (Figure 3). Subunit vaccines contain $M t b$ antigens expressed as recombinant proteins that are formulated with different adjuvants or expressed by recombinant viral vectors that are used as vehicles for the administration of antigens [47]. Most of the current subunit vaccine candidates are vaccines with limited antigen diversity which are designed to enhance prior immunity mediated by T cells [49] (Figure 3).

There is a total of $14 \mathrm{~TB}$ vaccine candidates in clinical trials today, seven are based on subunits and seven consist on whole-cell mycobacteria [47,50] (Figure 3). Of the subunit candidates, four are mycobacterial fusion protein(s) in new adjuvant formulations (ID93: GLA-SE, H56.IC31, M72:ASO1E, GamTBVac) and three are based on recombinant live-attenuated or replication-deficient virus-vectored expressing one or more Mtb proteins (Ad5Ag85, ChadOx1.85/MVA85A, TB/FLU-04L). Of the whole-cell mycobacterial candidates, there are four candidates based on inactivated/extracts of mycobacteria (M. vaccae, MIP, DAR-901, RUTI) and three vaccine candidates are based on live attenuated mycobacteria, one is BCG revaccination, since a positive signal has been seen in prevention studies of $M t b$ infection [51], other is based on the use of recombinant BCG (VPM1002) and the third is derived from rationally attenuated $M t b$ (MTBVAC).

\subsection{Whole-Cell Vaccine Candidates}

\subsubsection{Live Attenuated Vaccines}

Attenuated live vaccines based in $M t b$ in humans is expected to stimulate specific host-immune responses mimicking natural TB infection without causing disease (similar to with latent TB infection (LTBI)). In support of this rationale, prospective cohort studies with individuals exposed to patients with active TB indicate that those persons LTBI could be close to $80 \%$ more protected against secondary $M t b$ infection than individuals naïve to $M t b$ infection [52]. MTBVAC is based on the Pasteurian approach of vaccinology, to attenuate the pathogen from a human clinical isolate. The rational attenuation of MTBVAC is due to deletions in the major virulence genes phoP and fadD26. Following the Geneva consensus for attenuated TB vaccines [30], antibiotic markers were eliminated in MTBVAC [53]. As an attenuated derivative from the human pathogen, MTBVAC contains all antigens present in $M t b$ [54], including those contained in the RD1 region, which is deleted in BCG and responsible for increasing the protection in animal models [55] (Figure 3). The primary target population of MTBVAC are neonates; the secondary target population includes adolescents and adults (as a booster vaccine). A Phase Ia trial 
in adults showed that MTBVAC is safe and immunogenic [56] and a second Phase $\mathrm{Ib}$ in neonates in an endemic country showed that MTBVAC is as safe as BCG and more immunogenic [57]. Dose-defining Phase IIa trials in both target populations started in 2019 with funding support from US NIH in collaboration with IAVI in adults (ClinicalTrials.gov NCT02933281) and the EDCTP in collaboration with TBVI in neonates (ClinicalTrials.gov NCT03536117) (Figure 2). MTBVAC development is included in TBVI pipeline. MTBVAC was discovered and constructed at the University of Zaragoza (Spain) in collaboration with Pasteur Institute (France). Recently it was demonstrated that similarly to BCG, MTBVAC is able to induce trained immunity through the induction of glycolysis and glutaminolysis, accumulation of histone methylation marks at the promoters of pro-inflammatory genes, facilitating an enhanced response after secondary challenge. Importantly, these findings in human primary myeloid cells are complemented by a strong MTBVAC induced heterologous protection against a lethal challenge with Streptococcus pneumoniae in an experimental murine model of pneumonia (Tarancon et al. in press PLOS Pathogens 2020). The Spanish Biopharmaceutical company Biofabri is vaccine manufacturer of MTBVAC responsible for industrial and clinical development of the vaccine under the umbrella of TBVI.

BCG/BCG revaccination strategy showed a positive signal in prevention studies of $M t b$ infection [51] and has been included for application in adolescents and adults vaccinated with BCG at birth (Figure 2). BCG revaccination in adults reduced the rate of upper respiratory tract infections as compared to a subunit vaccine or placebo groups ( $2.1 \%, 9.4 \%$ and $7.9 \%$, respectively; $\mathrm{P}<0.001$ for both comparisons) [51] suggesting that BCG revaccination could prevent respiratory diseases including AMR forms of these diseases. The POI trial tested the ability of BCG revaccination to prevent $M t b$ infection using IGRA conversion QuantiFERON test in healthy South African adolescents [51]. However, considering the difficulty of understanding what IGRA conversion and reversion means in terms of developing TB disease, POD trials should be performed in order to obtain the authorization by the regulatory agencies for licensure of new TB vaccines. BCG/BCG revaccination strategy is included in the pipe line of the Bill and Melinda Gates Foundation.

VPM1002 is a recombinant M. bovis BCG, which is developed by the Max Planck Institute in Berlin to express listeriolysin from Listeria monocytogenes and with a deletion of the gene coding for urease C [58] (Figure 3). VPM1002 is in clinical development led by Vakzine Projekt Management and in collaboration with Serum Institute of India and is included in TBVI pipeline. The rationale is to improve the effectiveness of BCG by inserting additional genes. Two Phase I trials for safety and immunogenicity in adults and newborns have been published $[59,60]$ and a Phase Ilb efficacy trial is currently being carried out in South Africa to assess the safety and immunogenicity and protective efficacy of the vaccine including uninfected HIV-exposed newborns, with support by EDCTP. Other clinical trials undergoing with VPM1002 include a phase II/III POR and a Phase III POD trial in India [61] (Figure 2).

\subsubsection{Inactivated Whole-Cell Mycobacteria}

Most of inactivated whole cell mycobacterial TB vaccine candidates have been designed as "therapeutic vaccines" seeking to reduce treatment length in people infected with latent TB, or reduce the likelihood of recurrence after the end of a treatment [48].

M. vaccae ${ }^{\mathrm{TM}}$ vaccine is a lysate comprised of inactivated Mycobacterium vaccae (non-tuberculous mycobacteria) developed as an immunotherapeutic agent to help shorten TB treatment for patients with drug-susceptible TB and licensed by the China Food and Drug Administration (FDA). A Phase III study of efficacy has been recently published [2,62] (Figures 2 and 3).

MIP Immuvac is a heat-killed Mycobacterium indicus pranii vaccine, approved by the drug controller general of India and FDA as an immune-therapeutic and immunoprophylactic strategy for use in multibacillary leprosy patients (as an adjunct to standard multidrug therapy), and for preventing the development of leprosy among close contacts of leprosy patients. A Phase III efficacy and safety 
trial for preventing pulmonary $\mathrm{TB}$ among healthy house-hold contacts of sputum smear-positive $\mathrm{TB}$ patients is underway in India [2] (Figure 2).

DAR-901 is based on a heat-inactivated non-tuberculous bacterium M. vaccae renamed as Mycobacterium obuense which was produced by former Aeras (Rockville, MD, USA) [63] for use as booster vaccine in BCG vaccines (Figure 3). It is the scalable manufacturing version of candidate vaccine SRL172, which showed efficacy in a Phase III trial among HIV-infected adults in Tanzania. DAR-901 is in a Phase IIb prevention of infection trial among BCG-primed adolescents, also in the United Republic of Tanzania. The trial is scheduled for completion in 2020 [2] (Figure 2).

RUTI@is a liposomal formulation containing fragmented, detoxified $M t b$ grown under stress conceived conditions, as a potential therapeutic vaccine (Figure 3). RUTI is sponsored by the Archivel Farma (Spain), and is currently in a Phase IIa of clinical trials [64,65] (Figure 2). When administered one month after isoniazid treatment, RUTI showed safety and immunogenicity in individuals with latent TB infection (LTBI). Plans for evaluation in a POD trial among HIV-infected and uninfected patients with LTBI are underway [47].

\subsection{Subunit Vaccines}

\subsubsection{Subunit Viral Vectored Vaccines}

There are three subunit vaccines that use attenuated viral vectors by different routes of administration.

Ad5HuAg85A vaccine, developed by the University of McMaster in Canada, consists of a human adenovirus serotype 5 vector that expresses Ag85A and is administered intramuscularly (Figure 3). AdHu5Ag85A has been evaluated in a Phase 1 safety and immunogenicity study in BCG-naïve and BCG-immunized healthy adults by the intramuscular route showing adequate safety and tolerability [47,66] (Figure 2).

ChAdOx85A/MVA85A vaccine strategy, developed by the University of Oxford, like the recombinant pox vaccine MVA85A (mentioned above), ChAdOx85A is a simian adenovirus which also expresses Ag85A (Figure 3). Both vaccine candidates are tested together in a joint heterologous prime-boost regimen delivered through both systemic and mucosal routes in BCG-vaccinated individuals. A Phase I trial of intramuscular administration of ChAdOx85A in BCG vaccinated adults in the United Kingdom, tested alone and as part of a prime-boost strategy with MVA85A, has been completed. Aerosol administration of ChAdOx185A is currently evaluated in a Phase I trial among BCG-vaccinated adults in Switzerland (Figure 2). Safety and immunogenicity of aerosol administration of MVA85A in BCG-vaccinated individuals and in people with a latent TB infection have been evaluated. In 2019, plans for a Phase IIa safety and immunogenicity trial among adults and adolescents in Uganda were underway with the aim of evaluating intramuscular administration of ChAdOx185A and MVA85A [2].

TB/FLU-04L is based on an attenuated replication-deficient influenza virus vector expressing antigens Ag85A and ESAT-6 (Figure 3). It was designed as a preventive booster vaccine in BCG-vaccinated infants, adolescents and adults. It is about to start a Phase Ila study in individuals with latent TB [2] (Figure 2).

\subsubsection{Adjuvanted Subunit Vaccines}

D93 + GLA-SE vaccine was developed by the Infectious Disease Research Institute (IDRI) in the United States. ID93+GLA-SE comprises four $M t b$ antigens, of which three are associated with virulence (Rv2608, Rv3619 and Rv3620) and one (Rv1813), with latency) formulated with the adjuvant GLA-SE for delivery [67] (Figure 3). It has been evaluated Phase IIa trial in South Africa among HIV-negative TB patients who have recently completed TB treatment for active pulmonary disease [68] (Figure 2). This is in preparation for Phase II safety and immunogenicity trials among adults undergoing active TB therapy. A POI Phase IIa trial in BCG-vaccinated healthy health care workers is also underway [2]. 
H56:IC31 is a fusion protein of three $M t b$ antigens (Ag85B, ESAT- 6 and Rv2660c) and delivered in the adjuvant IC31@ (Valneva Austria GmBH, Vienna, Austria) (Figure 3). H56:IC31 was discovered and developed by the Statem Serum Institut (SSI) of Copenhagen. It has been tested in three Phase I or I/IIa trials for safety and immunogenicity in BCG-vaccinated adults (Figure 2) showing acceptable safety and immunogenicity. It has completed a Phase $\mathrm{Ib}$ safety and immunogenicity trial in adolescents. A POR Phase IIb trial funded by EDCTP and coordinated by IAVI is underway in South Africa and the United Republic of Tanzania [2].

M72/AS01E is also a subunit candidate vaccine comprising two $M t b$ antigens (32A and 39A) formulated in the AS01 adjuvant for delivery (Figure 3), also used in the formulation of the malaria vaccine (RTS, S/AS01, GlaxoSmithKline) and the recombinant zoster vaccine Shingrix, GlaxoSmithKline [69]. It was evaluated in a IIb efficacy trial in in Kenya, South Africa and Zambia among $M t b$-infected HIV-negative adults whose data showed $54.0 \%$ protective efficacy in $M t b$-infected young adult women [69] (Figure 2). The immunogenicity analysis after end of the three-year follow-up showed that M72/AS01 elicited an immune response and provided protection against progression to pulmonary TB disease for at least three years [70]. This is the first time a proof-of-principle trial demonstrates vaccine-induced protection against clinical TB disease. However, whether M72/AS01 could provide protection against TB among $M t b$-uninfected and HIV-negative individuals and in people from other geographical areas remain key questions to be answered. M72/AS01E has been exclusively licensed to the Medical Research Institute of Bill and Melinda Gates Foundation for further development.

GamTBvac is a fusion protein comprising Mtb antigens Ag85A and ESAT6-CFP-10 with the dextran-binding domain immobilized on dextran. It is formulated with an adjuvant consisting of a DEAE-dextran core and CpG oligodeoxynucleotides (TLR9 agonist) (Figure 3). GamTBvac is undergoing a Phase IIa safety and immunogenicity evaluation in healthy BCG-vaccinated adults, following a successful Phase 1 safety and immunogenicity trial in Russia [47] (Figure 2).

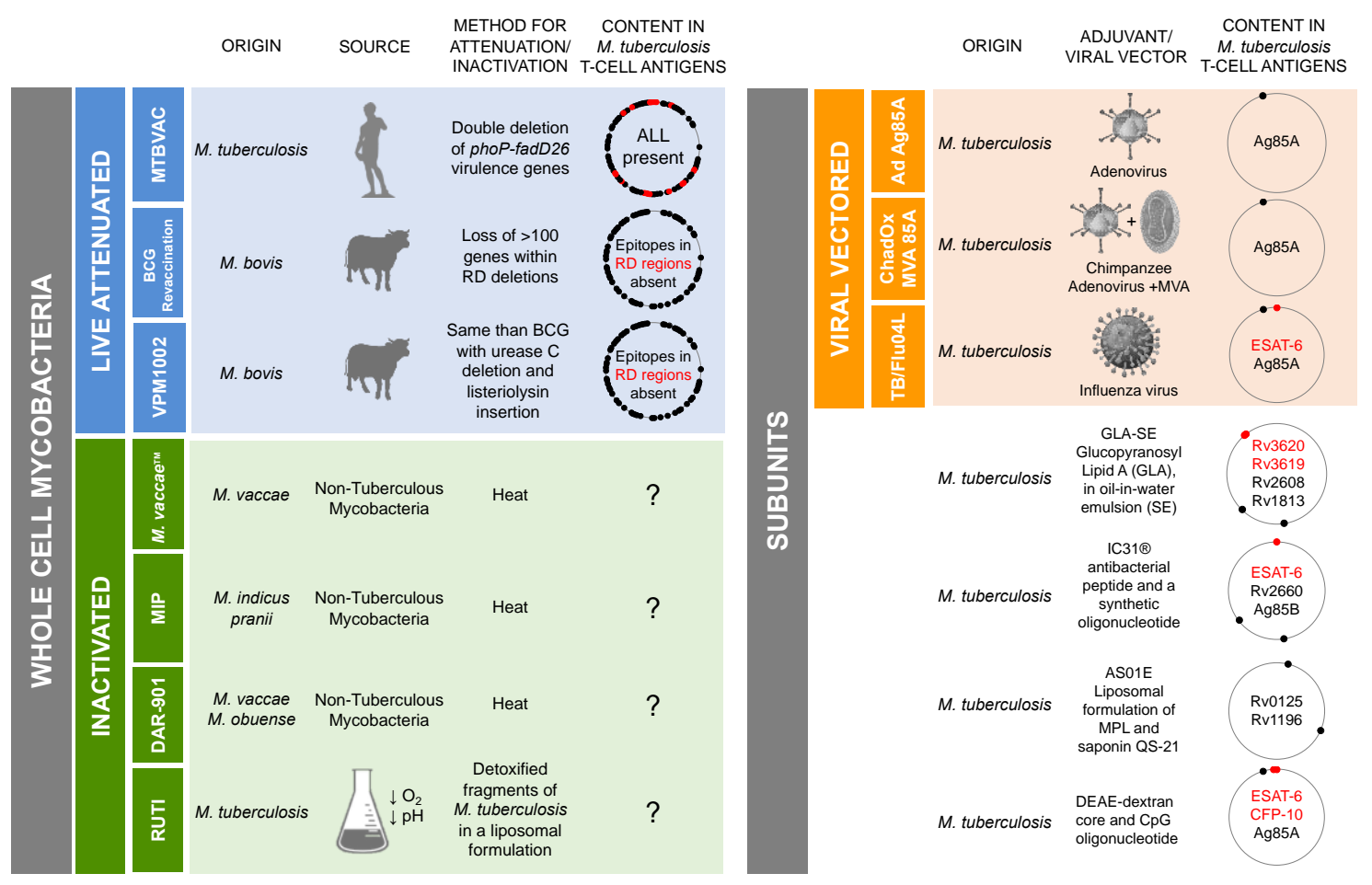

Figure 3. The diversity of TB vaccine candidates in clinical trials. Schematic table showing the main characteristics of the vaccine candidates. The table is coloured according to vaccine strategies indicated in Figure 2 and contains representative information for each candidate, including the mycobacterial origin from each vaccine and their antigenic content. 


\section{Target Population for a New TB Vaccine}

In its Preferred Product Characteristics (PPC) document for TB vaccines, WHO defines two target populations for which a new $\mathrm{TB}$ vaccine could play a very important role in the fight against TB including resistant forms of the disease [3,4]. The WHO prioritizes two types of strategies one, safe, effective and affordable TB vaccines for adolescents and adults and the other, a TB vaccine for neonates and infants with improved safety and efficacy as compared to BCG.

Following the negative results of the first efficacy study which was conducted with MVA5A in infants, the priority population was changed to mainly adults. Modelling studies suggest that new effective vaccines for adolescents and adults, who are responsible for disease transmission, would have the greatest impact in halting TB incidence [71,72]. These studies suggest that although disease incidence in children under five years of age is considerable, TB transmission among this age-group is not common [73].

Prophylactic prime vaccines should be compared to BCG, since whole-cell vaccines seek to protect better than BCG. The immune responses elicited by live mycobacterial vaccines are considered to be specific and long-lasting, and these responses are not obtained with subunit vaccines. Vaccine persistence or restricted replication in vivo could account for the differences of immune responses, as observed for other live human vaccines, such as polio, measles, and yellow fever [48]. Considering the variability in BCG formulations, new TB vaccine trials which use BCG as reference comparator, should be interpreted cautiously with reference to a specific BCG formulation avoiding generalization of data to all BCGs [13].

Since the majority of the adolescent and adult population in countries endemic of TB has been previously vaccinated with BCG at birth, what is sought with subunit candidates comprising specific $M t b$ antigens is to potentiate the pre-existing immunity induced by BCG $[2,46,62]$. Recent studies indicate that new TB vaccines that are compared to BCG should be interpreted cautiously with reference to a specific BCG formulation and not presumed to generalize to all BCGs [13]. In addition, testing the new concepts of vaccines in relevant animal models such as NHP, should be key before advancing into expensive clinical trials of efficacy [74,75]. Something that has been questioned in the MVA85A efficacy study [38] was that the efficacy experiments in NHP, which showed lack of efficacy by the tested clinical route and dose of administration [76,77]. We should be very careful not to repeat mistakes with new candidates. Recently, it was published in NHP, that boosting BCG with M72/ASO1E or H56/CAF01or rAd5 failed to enhance BCG-induced protection against TB [78]. Thus, care should be taken with the advanced clinical development of such candidates.

Healthy newborns represent the most sensitive population without pre-existing immunity to BCG or environmental mycobacteria, which in older groups can lead to possible effects of masking and blocking vaccination $[79,80]$. We, therefore, think that the efficacy should be studied in newborns as first step, and once the efficacy of a new vaccine has been established, then such new candidates should be tested for efficacy in adolescents and adults where the impact on TB will be greater given that the pulmonary forms are responsible for this transmission [80].

Author Contributions: Writing-review and editing, C.M., N.A., D.M. and J.G.-A. All authors have read and agreed to the published version of the manuscript.

Funding: This work has been funded by the Ministry of Science (RTI2018-097625-B-I00) and the European and Developing Countries Clinical Trials Partnership (EDCTP) RIA2016V-1637.

Acknowledgments: In this section, you can acknowledge any support given which is not covered by the author contribution or funding sections. This may include administrative and technical support, or donations in kind (e.g., materials used for experiments).

Conflicts of Interest: C.M., D.M., N.A. and J.G.-A. are inventors of patents related to the tuberculosis vaccine, the owner of which is the University of Zaragoza and of which the Spanish biotechnology company Biofabri is responsible for industrial and clinical development of MTBVAC. 


\section{References}

1. Bloom, D.E.; Black, S.; Salisbury, D.; Rappuoli, R. Antimicrobial resistance and the role of vaccines. Proc. Natl. Acad. Sci. USA 2018, 115, 12868-12871. [CrossRef] [PubMed]

2. WHO. Global Tuberculosis Report 2019; WHO, Ed.; WHO: Geneva, Switzerland, 2019; pp. 1-297.

3. WHO. Preferred Product Characteristics for New Tuberculosis Vaccines; WHO: Geneva, Switzerland, 2018; pp. 1-28.

4. Schrager, L.K.; Chandrasekaran, P.; Fritzell, B.H.; Hatherill, M.; Lambert, P.-H.; Mcshane, H.; Tornieporth, N.; Vekemans, J. WHO preferred product characteristics for new vaccines against tuberculosis. Lancet Infect. Dis. 2018, 18, 828-829. [CrossRef]

5. Mangtani, P.; Abubakar, I.; Ariti, C.; Beynon, R.; Pimpin, L.; Fine, P.E.M.; Rodrigues, L.C.; Smith, P.G.; Lipman, M.; Whiting, P.F.; et al. Protection by BCG vaccine against tuberculosis: A systematic review of randomized controlled trials. Clin. Infect. Dis. 2014, 58, 470-480. [CrossRef] [PubMed]

6. Zwerling, A.; Behr, M.A.; Verma, A.; Brewer, T.F.; Menzies, D.; Pai, M. The BCG World Atlas: A Database of Global BCG Vaccination Policies and Practices. PLoS Med. 2011, 8, e1001012. [CrossRef] [PubMed]

7. Fine, P.E. ScienceDirect.com-The Lancet-Variation in protection by BCG: Implications of and for heterologous immunity. Lancet 1995, 346, 1339-1345. [CrossRef]

8. Brosch, R.; Gordon, S.V.; Garnier, T.; Eiglmeier, K.; Frigui, W.; Valenti, P.; Santos, S.D.; Duthoy, S.; Lacroix, C.; Garcia-Pelayo, C.; et al. Genome plasticity of BCG and impact on vaccine efficacy. Proc. Natl. Acad. Sci. USA 2007, 104, 5596-5601. [CrossRef]

9. Gheorghiu, M.; Lagranderie, M.; Balazuc, A.-M. Vaccines: A Biography; Artenstein, A.W., Ed.; Springer New York: New York, NY, USA, 2009; pp. 125-140.

10. The BCG World Atlas Is a Database of Global BCG Vaccination Policies and Practices. Available online: http://www.bcgatlas.org (accessed on 1 April 2020).

11. The European Centre for Disease Prevention and Control Is an Agency of the European Union and Provides Access to the Vaccine Schedules in All Countries of the European Union. Available online: http://vaccine-schedule.ecdc.europa.eu/Pages/Scheduler.aspx (accessed on 1 April 2020).

12. Lambach, D.P. Who Informative Sheet BCG; WHO: Geneva, Switzerland, 2012; pp. 1-5.

13. Angelidou, A.; Conti, M.-G.; Diray-Arce, J.; Benn, C.S.; Shann, F.; Netea, M.G.; Liu, M.; Potluri, L.P.; Sanchez-Schmitz, G.; Husson, R.; et al. Licensed Bacille Calmette-Guérin (BCG) formulations differ markedly in bacterial viability, RNA content and innate immune activation. Vaccine 2020, 38, 2229-2240. [CrossRef]

14. Martinón-Torres, F.; Martin, C. Tuberculosis Vaccines. In Pediatric Vaccines and Vaccinations; Springer International Publishing: Cham, Switzerland, 2017; Volume 359, pp. 149-160.

15. Ho, M.M.; Southern, J.; Kang, H.-N.; Knezevic, I. WHO Informal Consultation on standardization and evaluation of BCG vaccines Geneva, Switzerland 22-23 September 2009. Vaccine 2010, 28, 6945-6950. [CrossRef]

16. Behr, M.A. BCG-different strains, different vaccines? Lancet Infect. Dis. 2002, 2, 86-92. [CrossRef]

17. Zhang, L.; Ru, H.-W.; Chen, F.-Z.; Jin, C.-Y.; Sun, R.-F.; Fan, X.-Y.; Guo, M.; Mai, J.-T.; Xu, W.-X.; Lin, Q.-X.; et al. Variable Virulence and Efficacy of BCG Vaccine Strains in Mice and Correlation with Genome Polymorphisms. Mol. Ther. 2016, 24, 398-405. [CrossRef] [PubMed]

18. Benn, C.S.; Netea, M.G.; Selin, L.K.; Aaby, P. A small jab—A big effect: Nonspecific immunomodulation by vaccines. Trends Immunol. 2013, 34, 431-439. [CrossRef] [PubMed]

19. Cauchi, S.; Locht, C. Non-specific Effects of Live Attenuated Pertussis Vaccine Against Heterologous Infectious and Inflammatory Diseases. Front. Immunol. 2018, 9, 2872. [CrossRef] [PubMed]

20. Kleinnijenhuis, J.J.; Quintin, J.J.; Preijers, F.F.; Joosten, L.A.B.L.; Ifrim, D.C.D.; Saeed, S.S.; Jacobs, C.C.; van Loenhout, J.J.; de Jong, D.D.; Stunnenberg, H.G.H.; et al. Bacille Calmette-Guerin induces NOD2-dependent nonspecific protection from reinfection via epigenetic reprogramming of monocytes. Proc. Natl. Acad. Sci. USA 2012, 109, 17537-17542. [CrossRef] [PubMed]

21. Ota, M.O.C.; Vekemans, J.; Schlegel-Haueter, S.E.; Fielding, K.; Sanneh, M.; Kidd, M.; Newport, M.J.; Aaby, P.; Whittle, H.; Lambert, P.-H.; et al. Influence of Mycobacterium bovis bacillus Calmette-Guérin on antibody and cytokine responses to human neonatal vaccination. J. Immunol. 2002, 168, 919-925. [CrossRef] 
22. de Castro, M.J.; Pardo-Seco, J.; Martinón-Torres, F. Nonspecific (Heterologous) Protection of Neonatal BCG Vaccination Against Hospitalization Due to Respiratory Infection and Sepsis. Clin. Infect. Dis. 2015, 60, 1611-1619. [CrossRef]

23. Iglesias, M.J.; Martin, C. Editorial Commentary: Nonspecific Beneficial Effects of BCG Vaccination in High-income Countries, Should We Extend Recommendation of BCG Vaccination? Clin. Infect. Dis. 2015, 60, 1620-1621. [CrossRef]

24. Netea, M.G.; Joosten, L.A.B.; Latz, E.; Mills, K.H.G.; Natoli, G.; Stunnenberg, H.G.; O’Neill, L.A.J.; Xavier, R.J. Trained immunity: A program of innate immune memory in health and disease. Science 2016, 352, aaf1098. [CrossRef]

25. Arts, R.J.W.; Moorlag, S.J.C.F.M.; Novakovic, B.; Li, Y.; Wang, S.-Y.; Oosting, M.; Kumar, V.; Xavier, R.J.; Wijmenga, C.; Joosten, L.A.B.; et al. BCG Vaccination Protects against Experimental Viral Infection in Humans through the Induction of Cytokines Associated with Trained Immunity. Cell Host Microbe 2018, 23, 89-100.e5. [CrossRef]

26. Arts, R.J.W.; Carvalho, A.; La Rocca, C.; Palma, C.; Rodrigues, F.; Silvestre, R.; Kleinnijenhuis, J.; Lachmandas, E.; Gonçalves, L.G.; Belinha, A.; et al. Immunometabolic Pathways in BCG-Induced Trained Immunity. Cell Rep. 2016, 17, 2562-2571. [CrossRef]

27. Walk, J.; de Bree, L.C.J.; Graumans, W.; Stoter, R.; van Gemert, G.-J.; van de Vegte-Bolmer, M.; Teelen, K.; Hermsen, C.C.; Arts, R.J.W.; Behet, M.C.; et al. Outcomes of controlled human malaria infection after BCG vaccination. Nat. Commun. 2019, 10, 874-878. [CrossRef]

28. Berendsen, M.L.; van Gijzel, S.W.; Smits, J.; de Mast, Q.; Aaby, P.; Benn, C.S.; Netea, M.G.; van der Ven, A.J. BCG vaccination is associated with reduced malaria prevalence in children under the age of 5 years in sub-Saharan Africa. BMJ Glob. Health 2019, 4, e001862-11. [CrossRef] [PubMed]

29. Walker, K.B.; Brennan, M.J.; Ho, M.M.; Eskola, J.; Thiry, G.; Sadoff, J.; Dobbelaer, R.; Grode, L.; Liu, M.A.; Fruth, U.; et al. The second Geneva Consensus: Recommendations for novel live TB vaccines. Vaccine 2010, 28, 2259-2270. [CrossRef] [PubMed]

30. Kamath, A.T.; Fruth, U.; Brennan, M.J.; Dobbelaer, R.; Hubrechts, P.; Ho, M.M.; Mayner, R.E.; Thole, J.; Walker, K.B.; Liu, M.; et al. AERAS Global TB Vaccine Foundation; World Health Organization New live mycobacterial vaccines: The Geneva consensus on essential steps towards clinical development. Vaccine 2005, 23, 3753-3761. [CrossRef] [PubMed]

31. Rappuoli, R.; Aderem, A. A 2020 vision for vaccines against HIV, tuberculosis and malaria. Nature 2011, 473, 463-469. [CrossRef]

32. Marinova, D.; Gonzalo-Asensio, J.; Aguilo, N.; Martin, C. Recent developments in tuberculosis vaccines. Expert Rev. Vaccines 2013, 12, 1431-1448. [CrossRef] [PubMed]

33. FMedSci, H.M. Tuberculosis 20193 Insights and challenges in tuberculosis vaccine development. Lancet Respir. Med. 2019, 7, 810-819.

34. On 1st Oct 2018, The International Vaccine Initiative (IAVI) https://www.iavi.org/ and Aeras, a Nonprofit Organization Dedicated to Developing Tuberculosis (TB) Vaccines, Today Announced the Transfer to IAVI of Aeras' TB Vaccine Clinical Research Programs and Assets, Consisting of Certain Clinical Staff, Clinical Programs, Biorepository, Funding Commitments, and Other Assets. The Transaction, Effective Today, Will Enable the Continuity of Aeras' Core TB Vaccine Clinical Programs and Will Expand IAVI's Clinical Development Capabilities and Network, Incorporating an Experienced Clinical Team and South African Clinical Partner Network with a Strong Track Record in Later-Stage Clinical Trials and Work with Adolescent and Adult Populations. Available online: https://www.iavi.org/newsroom/press-releases/2018/iavi-acquiresaeras-tb-vaccine-clinical-programs-and-assets (accessed on 1 April 2020).

35. Kaufmann, S.H.E.; Dockrell, H.M.; Drager, N.; Ho, M.M.; Mcshane, H.; Neyrolles, O.; Ottenhoff, T.H.M.; Patel, B.; Roordink, D.; Spertini, F.; et al. TBVAC2020 Consortium TBVAC2020: Advancing Tuberculosis Vaccines from Discovery to Clinical Development. Front. Immunol. 2017, 8, 1203. [CrossRef]

36. The TuBerculosis Vaccine Initiative (TBVI) Is a Non-Profit Foundation that Facilitates the Discovery and Development of New, Safe and Effective TB Vaccines that Are Accessible and Affordable for All People. Available online: https://www.tbvi.eu/ (accessed on 1 April 2020). 
37. The European \& Developing Countries Clinical Trials Partnership (EDCTP) Funds Clinical Research to Accelerate the Development of New or Improved Drugs, Vaccines, Microbicides and Diagnostics against HIV/AIDS, Tuberculosis and Malaria as well as Other Poverty-Related Infectious Diseases in sub-Saharan Africa, with a Focus on Phase II and III Clinical Trials. Available online: https://www.edctp.org/ (accessed on 1 April 2020).

38. Darrah, P.A.; Zeppa, J.J.; Maiello, P.; Hackney, J.A.; Wadsworth, M.H.; Hughes, T.K.; Pokkali, S.; Swanson, P.A.; Grant, N.L.; Rodgers, M.A.; et al. Prevention of tuberculosis in macaques after intravenous BCG immunization. Nature 2019, 577, 95-102. [CrossRef]

39. Dijkman, K.; Sombroek, C.C.; Vervenne, R.A.W.; Hofman, S.O.; Boot, C.; Remarque, E.J.; Kocken, C.H.M.; Ottenhoff, T.H.M.; Kondova, I.; Khayum, M.A.; et al. Prevention of tuberculosis infection and disease by local BCG in repeatedly exposed rhesus macaques. Nat. Med. 2019, 25, 255-262. [CrossRef]

40. Sander, P.; Clark, S.; Petrera, A.; Vilaplana, C.; Meuli, M.; Selchow, P.; Zelmer, A.; Mohanan, D.; Andreu, N.; Rayner, E.; et al. Deletion of zmp1 improves Mycobacterium bovis BCG-mediated protection in a guinea pig model of tuberculosis. Vaccine 2015, 33, 1353-1359. [CrossRef]

41. Gröschel, M.I.; Sayes, F.; Shin, S.J.; Frigui, W.; Pawlik, A.; Orgeur, M.; Canetti, R.; Honoré, N.; Simeone, R.; van der Werf, T.S.; et al. Recombinant BCG Expressing ESX-1 of Mycobacterium marinum Combines Low Virulence with Cytosolic Immune Signaling and Improved TB Protection. Cell Rep. 2017, 18, 2752-2765. [CrossRef] [PubMed]

42. Kroesen, V.M.; Madacki, J.; Frigui, W.; Sayes, F.; Brosch, R. Mycobacterial virulence: Impact on immunogenicity and vaccine research. F1000Res 2019, 8, 2025. [CrossRef]

43. Tameris, M.D.; Hatherill, M.; Landry, B.S.; Scriba, T.J.; Snowden, M.A.; Lockhart, S.; Shea, J.E.; McClain, J.B.; Hussey, G.D.; Hanekom, W.A.; et al. Safety and efficacy of MVA85A, a new tuberculosis vaccine, in infants previously vaccinated with BCG: A randomised, placebo-controlled phase $2 \mathrm{~b}$ trial. Lancet 2013, 381, 1021-1028. [CrossRef]

44. Tameris, M.; Mcshane, H.; McClain, J.B.; Landry, B.; Lockhart, S.; Luabeya, A.K.K.; Geldenhuys, H.; Shea, J.; Hussey, G.; van der Merwe, L.; et al. Lessons learnt from the first efficacy trial of a new infant tuberculosis vaccine since BCG. Tuberculosis 2013, 93, 143-149. [CrossRef]

45. Andrews, J.R.; Nemes, E.; Tameris, M.; Landry, B.S.; Mahomed, H.; McClain, J.B.; Fletcher, H.A.; Hanekom, W.A.; Wood, R.; Mcshane, H.; et al. Serial QuantiFERON testing and tuberculosis disease risk among young children: An observational cohort study. Lancet Respir. Med. 2017, 5, 282-290. [CrossRef]

46. Verver, S.; Warren, R.M.; Beyers, N.; Richardson, M.; van der Spuy, G.D.; Borgdorff, M.W.; Enarson, D.A.; Behr, M.A.; Van Helden, P.D. Rate of reinfection tuberculosis after successful treatment is higher than rate of new tuberculosis. Am. J. Respir. Crit. Care Med. 2005, 171, 1430-1435. [CrossRef]

47. Sable, S.B.; Posey, J.E.; Scriba, T.J. Tuberculosis Vaccine Development: Progress in Clinical Evaluation. Clin. Microbiol. Rev. 2019, 33, 16076. [CrossRef]

48. Scriba, T.J.; Kaufmann, S.H.E.; Henri Lambert, P.; Sanicas, M.; Martin, C.; Neyrolles, O. Vaccination Against Tuberculosis with Whole-Cell Mycobacterial Vaccines. J. Infect. Dis. 2016, 214, 659-664. [CrossRef]

49. Marinova, D.; Gonzalo-Asensio, J.; Aguilo, N.; Martin, C. MTBVAC from discovery to clinical trials in tuberculosis-endemic countries. Expert Rev. Vaccines 2017, 16, 565-576. [CrossRef]

50. TBVI is Continuously Working on the Development of New Vaccine Candidates. The Current TB Vaccine Pipeline (Last Update October 2019), Is as Follows. Available online: https://www.tbvi.eu/what-we-do/ pipeline-of-vaccines/ (accessed on 1 April 2020).

51. Nemes, E.; Geldenhuys, H.; Rozot, V.; Rutkowski, K.T.; Ratangee, F.; Bilek, N.; Mabwe, S.; Makhethe, L.; Erasmus, M.; Toefy, A.; et al. C-040-404 Study Team Prevention of M. tuberculosis Infection with H4:IC31 Vaccine or BCG Revaccination. N. Engl. J. Med. 2018, 379, 138-149. [CrossRef]

52. Andrews, J.R.; Noubary, F.; Walensky, R.P.; Cerda, R.; Losina, E.; Horsburgh, C.R. Risk of progression to active tuberculosis following reinfection with Mycobacterium tuberculosis. Clin. Infect. Dis. 2012, 54, 784-791. [CrossRef]

53. Arbués, A.; Aguilo, J.I.; Gonzalo-Asensio, J.; Marinova, D.; Uranga, S.; Puentes, E.; Fernandez, C.; Parra, A.; Cardona, P.-J.; Vilaplana, C.; et al. Construction, characterization and preclinical evaluation of MTBVAC, the first live-attenuated M. tuberculosis-based vaccine to enter clinical trials. Vaccine 2013, 31, 4867-4873.

54. Aguilo, N. MTBVAC: Attenuating the Human Pathogen of Tuberculosis (TB) Toward a Promising Vaccine against the TB Epidemic. Front. Immunol. 2017, 8, 1803. 
55. Aguilo, N.; Gonzalo-Asensio, J.; Alvarez-Arguedas, S.; Marinova, D.; Gomez, A.B.; Uranga, S.; Spallek, R.; Singh, M.; Audran, R.; Spertini, F.; et al. Reactogenicity to major tuberculosis antigens absent in BCG is linked to improved protection against Mycobacterium tuberculosis. Nat. Commun. 2017, 8, 16085. [CrossRef] [PubMed]

56. Spertini, F.; Audran, R.; Chakour, R.; Karoui, O.; Steiner-Monard, V.; Thierry, A.-C.; Mayor, C.E.; Rettby, N.; Jaton, K.; Vallotton, L.; et al. Safety of human immunisation with a live-attenuated Mycobacterium tuberculosis vaccine: A randomised, double-blind, controlled phase I trial. Lancet Respir. Med. 2015, 3, 953-962. [CrossRef]

57. Tameris, M.; Mearns, H.; Penn-Nicholson, A.; Gregg, Y.; Bilek, N.; Mabwe, S.; Geldenhuys, H.; Shenje, J.; Luabeya, A.K.-K.; Murillo, I.; et al. Live-attenuated Mycobacterium tuberculosis vaccine MTBVAC versus BCG in adults and neonates: A randomised controlled, double-blind dose-escalation trial. Lancet Respir. Med. 2019, 7, 757-770. [CrossRef]

58. Grode, L. Increased vaccine efficacy against tuberculosis of recombinant Mycobacterium bovis bacille Calmette-Guerin mutants that secrete listeriolysin. J. Clin. Investig. 2005, 115, 2472-2479. [CrossRef]

59. Grode, L.; Ganoza, C.A.; Brohm, C.; Weiner, J., 3rd; Eisele, B.; Kaufmann, S.H.E. Safety and immunogenicity of the recombinant BCG vaccine VPM1002 in a phase 1 open-label randomized clinical trial. Vaccine 2013, 31, 1340-1348. [CrossRef]

60. Loxton, A.G.; Knaul, J.K.; Grode, L.; Gutschmidt, A.; Meller, C.; Eisele, B.; Johnstone, H.; van der Spuy, G.; Maertzdorf, J.; Kaufmann, S.H.E.; et al. Safety and Immunogenicity of the Recombinant Mycobacterium bovis BCG Vaccine VPM1002 in HIV-Unexposed Newborn Infants in South Africa. Clin. Vaccine Immunol. 2017, 24, e00439-16. [CrossRef]

61. Frick, M. TB VACCINES Pipeline Report 2019; Treatment Action Group, TAG: New York, NY, USA, 2019; pp. 1-25.

62. Bourinbaiar, A.S.; Batbold, U.; Efremenko, Y.; Sanjagdorj, M.; Butov, D.; Damdinpurev, N.; Grinishina, E.; Mijiddorj, O.; Kovolev, M.; Baasanjav, K.; et al. Phase III, placebo-controlled, randomized, double-blind trial of tableted, therapeutic TB vaccine (V7) containing heat-killed M. vaccae administered daily for one month. J. Clin. Tuberc. Other Mycobact. Dis. 2020, 18, 100141. [CrossRef]

63. Ginsberg, A.M.; Ruhwald, M.; Mearns, H.; Mcshane, H. TB vaccines in clinical development. Tuberculosis 2016, 99, S16-S20. [CrossRef] [PubMed]

64. Vilaplana, C.; Montané, E.; Pinto, S.; Barriocanal, A.M.; Domenech, G.; Torres, F.; Cardona, P.-J.; Costa, J. Double-blind, randomized, placebo-controlled Phase I Clinical Trial of the therapeutical antituberculous vaccine RUTI ${ }^{\circledR}$. Vaccine 2010, 28, 1106-1116. [CrossRef] [PubMed]

65. Nell, A.S.; D’lom, E.; Bouic, P.; Sabaté, M.; Bosser, R.; Picas, J.; Amat, M.; Churchyard, G.; Cardona, P.-J. Safety, Tolerability, and Immunogenicity of the Novel Antituberculous Vaccine RUTI: Randomized, Placebo-Controlled Phase II Clinical Trial in Patients with Latent Tuberculosis Infection. PLoS ONE 2014, 9, e89612. [CrossRef] [PubMed]

66. Smaill, F.; Jeyanathan, M.; Smieja, M.; Medina, M.F.; Thanthrige-Don, N.; Zganiacz, A.; Yin, C.; Heriazon, A.; Damjanovic, D.; Puri, L.; et al. A Human Type 5 Adenovirus-Based Tuberculosis Vaccine Induces Robust T Cell Responses in Humans Despite Preexisting Anti-Adenovirus Immunity. Sci. Transl. Med. 2013, 5, 205ra134. [CrossRef] [PubMed]

67. Coler, R.N.; Day, T.A.; Ellis, R.; Piazza, F.M.; Beckmann, A.M.; Vergara, J.; Rolf, T.; Lu, L.; Alter, G.; Hokey, D.; et al. TBVPX-113 Study Team The TLR-4 agonist adjuvant, GLA-SE, improves magnitude and quality of immune responses elicited by the ID93 tuberculosis vaccine: First-in-human trial. NPJ Vaccines 2018, 3, 34. [CrossRef]

68. Penn-Nicholson, A.; Tameris, M.; Smit, E.; Day, T.A.; Musvosvi, M.; Jayashankar, L.; Vergara, J.; Mabwe, S.; Bilek, N.; Geldenhuys, H.; et al. TBVPX-114 study team Safety and immunogenicity of the novel tuberculosis vaccine ID93 + GLA-SE in BCG-vaccinated healthy adults in South Africa: A randomised, double-blind, placebo-controlled phase 1 trial. Lancet Respir. Med. 2018, 6, 287-298. [CrossRef]

69. Van Der Meeren, O.; Hatherill, M.; Nduba, V.; Wilkinson, R.J.; Muyoyeta, M.; Van Brakel, E.; Ayles, H.M.; Henostroza, G.; Thienemann, F.; Scriba, T.J.; et al. Phase 2b Controlled Trial of M72/AS01E Vaccine to Prevent Tuberculosis. N. Engl. J. Med. 2018, 379, 1621-1634. [CrossRef]

70. Tait, D.R.; Hatherill, M.; Van Der Meeren, O.; Ginsberg, A.M.; Van Brakel, E.; Salaun, B.; Scriba, T.J.; Akite, E.J.; Ayles, H.M.; Bollaerts, A.; et al. Final Analysis of a Trial of M72/AS01E Vaccine to Prevent Tuberculosis. N. Engl. J. Med. 2019, 381, 2429-2439. [CrossRef] 
71. Yates, T.A.; Khan, P.Y.; Knight, G.M.; Taylor, J.G.; McHugh, T.D.; Lipman, M.; White, R.G.; Cohen, T.; Cobelens, F.G.; Wood, R.; et al. The transmission of Mycobacterium tuberculosis in high burden settings. Lancet Infect. Dis. 2016, 16, 227-238. [CrossRef]

72. Tovar, M.; Arregui, S.; Marinova, D.; Martin, C.; Sanz, J.; Moreno, Y. Bridging the gap between efficacy trials and model-based impact evaluation for new tuberculosis vaccines. Nat. Commun. 2019, 10, 5457. [CrossRef]

73. Blaser, N.; Zahnd, C.; Hermans, S.; Salazar-Vizcaya, L.; Estill, J.; Morrow, C.; Egger, M.; Keiser, O.; Wood, R. Tuberculosis in Cape Town: An age-structured transmission model. Epidemics 2016, 14, 54-61. [CrossRef] [PubMed]

74. Kashangura, R.; Sena, E.S.; Young, T.; Garner, P. Effects of MVA85A vaccine on tuberculosis challenge in animals: Systematic review. Int. J. Epidemiol. 2015, 44, 1970-1981. [CrossRef] [PubMed]

75. Godlee, F. We need better animal research, better reported. BMJ 2018, k124. [CrossRef]

76. Cohen, D. Oxford vaccine study highlights pick and mix approach to preclinical research. BMJ 2018. [CrossRef]

77. Macleod, M. Learning lessons from MVA85A, a failed booster vaccine for BCG. BMJ 2018. [CrossRef] [PubMed]

78. Darrah, P.A.; DiFazio, R.M.; Maiello, P.; Gideon, H.P.; Myers, A.J.; Rodgers, M.A.; Hackney, J.A.; Lindenstrøm, T.; Evans, T.; Scanga, C.A.; et al. Boosting BCG with proteins or rAd5 does not enhance protection against tuberculosis in rhesus macaques. NPJ Vaccines 2019, 4, 21. [CrossRef]

79. Barreto, M.L.; Pilger, D.; Pereira, S.M.; Genser, B.; Cruz, A.A.; Cunha, S.S.; Sant'Anna, C.; Hijjar, M.A.; Ichihara, M.Y.; Rodrigues, L.C. Causes of variation in BCG vaccine efficacy: Examining evidence from the BCG REVAC cluster randomized trial to explore the masking and the blocking hypotheses. Vaccine 2014, 32, 3759-3764. [CrossRef]

80. Arregui, S.; Sanz, J.; Marinova, D.; Martin, C.; Moreno, Y. On the impact of masking and blocking hypotheses for measuring the efficacy of new tuberculosis vaccines. Peer] 2016, 4, e1513. [CrossRef]

(C) 2020 by the authors. Licensee MDPI, Basel, Switzerland. This article is an open access article distributed under the terms and conditions of the Creative Commons Attribution (CC BY) license (http://creativecommons.org/licenses/by/4.0/). 